\title{
ON FULLY COUPLED THERMO-ELASTO-PLASTIC STOCHASTIC FINITE ELEMENT ANALYSIS OF STEEL STRUCTURES
}

\author{
Marcin Kamiński $^{1 *}$, Michał Strąkowski ${ }^{1}$ \\ ${ }^{1}$ Lodz University of Technology \\ Department of Structural Mechanics \\ Al. Politechniki 6, 90-924 Lodz, Poland \\ Marcin.Kaminski@p.lodz.pl
}

Keywords: Stochastic Finite Element Method, Fire simulation, Fully coupled thermal-stress analysis.

\begin{abstract}
This work demonstrates an application of the generalized stochastic perturbation technique and the corresponding Stochastic Finite Element Method in numerical analysis of the stresses for the steel structural element subjected to higher temperatures. This approach is based on higher order Taylor expansion of the input random parameters and of the resulting state functions and, independently, on the Least Squares Method determination of the structural responses to the input random parameters. An initial temperature equivalent to the fire exposure of the steel structure is taken as the input Gaussian random variable and uniquely defined by the constant mean value at outer surfaces of this element, where material parameters of the steel as Young modulus, yield strength, heat conductivity, capacity and thermal elongation are all considered as highly temperature-dependent. These functions are adopted according to the experimental evidence provided in the literature. We adopt various degree of polynomial functions to verify an influence of the response function choice upon the resulting probabilistic characteristics. This study is an example of a hybrid usage of the FEM system ABAQUS and the computer algebra system MAPLE in stochastic transient fully coupled thermo-mechanical analysis, where up to the fourth order probabilistic characteristics of the temperatures, displacements and stresses may be directly used in fire reliability analysis of the civil engineering structures with the temperature both independent and dependent material characteristics. The results obtained in this study may serve directly in the stochastic reliability analysis of the steel structures subjected to a fire according to both First and Second Order Reliability Methods (FORM and SORM).
\end{abstract}




\section{INTRODUCTION}

Reliability of engineering structures under fire conditions remains always very challenging and practically important knowledge. It is possible to use it for an efficient prediction of the failure time for the structures to get specific information about their fire resistance and evacuation time. The well-known Monte-Carlo simulation needs huge computer time effort. In this case it is better to use higher order perturbation techniques implemented with the FEM originating from the Second Order Second Moment method [1] relevant to small initial random fluctuations. We compute probabilistic coefficients of up to the fourth order to gain more information about probability density function itself. The basic probabilistic moments as well as skewness and kurtosis are calculated by full symbolic expansion of integral definitions and partial differentiation of the nodal response functions with respect to given random input variable. In this case we consider strength test of elasto-plastic steel cylindrical specimen subjected to high temperatures. All physical and mechanical steel properties like Young modulus, yield strength, conductivity, heat capacity and thermal elongation are temperature-dependent.

\section{THEORETICAL BACKGROUND}

Let us introduce the random variable $\mathrm{b}$ and its probability density function as $p_{b}(x)$. Then, the first two probabilistic moments of this variable are defined as [2]

$$
E[b] \equiv b^{0}=\int_{-\infty}^{+\infty} b p_{b}(x) d x,
$$

where $b^{0}$ means the average value of $b$ itself and

$$
\operatorname{Var}[b]=\int_{-\infty}^{+\infty}[b-E(b)]^{2} p_{b}(x) d x .
$$

Higher probabilistic moments and related coefficients may be defined according to the classical definitions from the probability theory. The basic idea of the stochastic perturbation approach employed here is to expand all input random variables and all the resulting state functions of the given boundary initial problem via Taylor series about their spatial expectations using the perturbation parameter $\varepsilon$. The random function $f$ with respect to its parameter $b$ around its mean value is given as follows

$$
f=f^{0}+\sum_{n=10}^{1} \frac{\varepsilon^{n}}{n !} \frac{\partial^{n} f}{\partial b^{n}} \Delta b^{n},
$$

where

$$
\varepsilon \Delta b=\varepsilon\left(b-b^{0}\right)
$$

is the first variation of variable $b$ about its expected value and symbol $(.)^{0}$ represents the value of a function calculated at its mean. Let us analyse further the expected values of stress state function $\sigma(b)$ defined analogously to the Eqn. 3 by its expansion via Taylor series as follows

$$
E[\sigma(b)]=\int_{-\infty}^{+\infty} \sigma(b) p_{b}(x) d x=\int_{-\infty}^{+\infty}\left(\sigma^{0}\left(b^{0}\right)+\sum_{n=10}^{1} \frac{\varepsilon^{n}}{n !} \frac{\partial^{n} \sigma(b)}{\partial b^{n}} \Delta b^{n}\right) p_{b}(x) d x .
$$


We remind that this power expansion is valid only if the state function is analytic in $\varepsilon$ and the series converge and, therefore, any criteria of convergence should include the magnitude of the perturbation parameter; perturbation parameter is taken as equal 1 in several practical computations and also here [3,4]. It yields for the input random variable with symmetric probability density function in the second order perturbation approach

$$
E[\sigma(b)]=\sigma^{0}\left(b^{0}\right)+\frac{1}{2 !} \frac{\partial^{2} \sigma(b)}{\partial b^{2}} \mu_{2}(b)+\cdots+\frac{1}{10 !} \frac{\partial^{10} \sigma(b)}{\partial b^{10}} \mu_{10}(b),
$$

where $\mu_{n}(b)$ denotes nth order central probabilistic moment of the variable $b$. All the terms with odd orders are equal to 0 for the symmetric random variable and the orders higher than the 10th one are neglected additionally. Similar considerations leads to the expressions, like the variance, for instance

$$
\operatorname{Var}[\sigma(b)]=\mu_{2}(\sigma(b))=\int_{-\infty}^{+\infty}(\sigma(b)-E[\sigma(b)])^{2} p(b) d b .
$$

Quite similarly, it is possible to derive 3rd central probabilistic moments using also the 10th order approach as

$$
\mu_{3}(\sigma(b))=\int_{-\infty}^{+\infty}(\sigma(b)-E[\sigma(b)])^{3} p(b) d b,
$$

and also the 4th order probabilistic moment may be recovered from this scheme as

$$
\mu_{4}(\sigma(b))=\int_{-\infty}^{+\infty}(\sigma(b)-E[\sigma(b)])^{4} p(b) d b .
$$

Let us mention that it is necessary to multiply each of these equations by the relevant order probabilistic moments of the input random variable to get the algebraic form convenient for any symbolic computations. Based on the classical definition of the variance we can calculate the coefficient of variation as fallows

$$
\alpha(\sigma(b))=\frac{\sqrt{\mu_{2}(\sigma(b))}}{E[\sigma(b)]} .
$$

Higher order moments and characteristics are determined to recognize the type of probabilistic distribution od the state functions. We compute in turn the skewness coefficients as the ratio of the third central probabilistic moment and the third power of standard deviation as

$$
S(\sigma(b))=\frac{\mu_{3}(\sigma(b))}{\left[\sqrt{\left.\mu_{2}(\sigma(b))\right]^{3}}\right.}
$$

and kurtosis as

$$
\kappa(\sigma(b))=\frac{\mu_{4}(\sigma(b))}{\left[\sqrt{\left.\mu_{2}(\sigma(b))\right]^{4}}\right.}-3 .
$$

It should be mentioned that at this stage the proposed procedure is independent from a choice of the initial probability distribution function, however a satisfactory probabilistic conver- 
gence of the final results may demand various lengths of the expansions for random variables with different distributions.

The method is based on the iterative coupled deterministic FEM [5] equations as follows

$$
\left\{\begin{array}{l}
\mathbf{C}^{(\alpha)} \boldsymbol{\theta}^{(\alpha)}+\hat{\mathbf{K}}^{(\alpha)} \boldsymbol{\theta}^{(\alpha)}=\hat{\mathbf{R}}^{(\alpha)} \\
\mathbf{K}^{(\alpha)}(\boldsymbol{\theta}) \mathbf{q}^{(\alpha)}(\boldsymbol{\theta})=\mathbf{R}^{(\alpha)}
\end{array},\right.
$$

where $\mathbf{C}$ is heat capacity matrix, $\boldsymbol{\theta}$ is nodal temperatures vector, $\mathbf{K}$ is heat conductivity matric, $\mathbf{K}$ denotes stiffness matrix, $\mathbf{R}$ is matrix of nodal loads, $\hat{\mathbf{R}}$ is a matrix of thermal loads and $\mathbf{q}$ is the solution displacement vector and $\alpha$ indices the test number relevant to Response Function Method [6]. We solve Eq. (13) in ABAQUS system. Further determination of the probabilistic moments from Eqs. $(5 \div 12)$ thanks to the symbolic derivation of all partial derivatives with respect to the given random variable $b$. We carry out all these calculations in computer algebra system MAPLE.

\section{NUMERICAL EXAMPLE}

Numerical example consist of the necking of an axi-symmetric steel specimen (Fig. 1). Its initial length is $2 L_{0}$ and initial radius is $R_{0}$ and $L_{0} / R_{0}=4$. We make small cut of its corner $\Delta R=0,005 R_{0} * 0.05 \mathrm{~mm}$ to ensure necking in the middle of it [7].
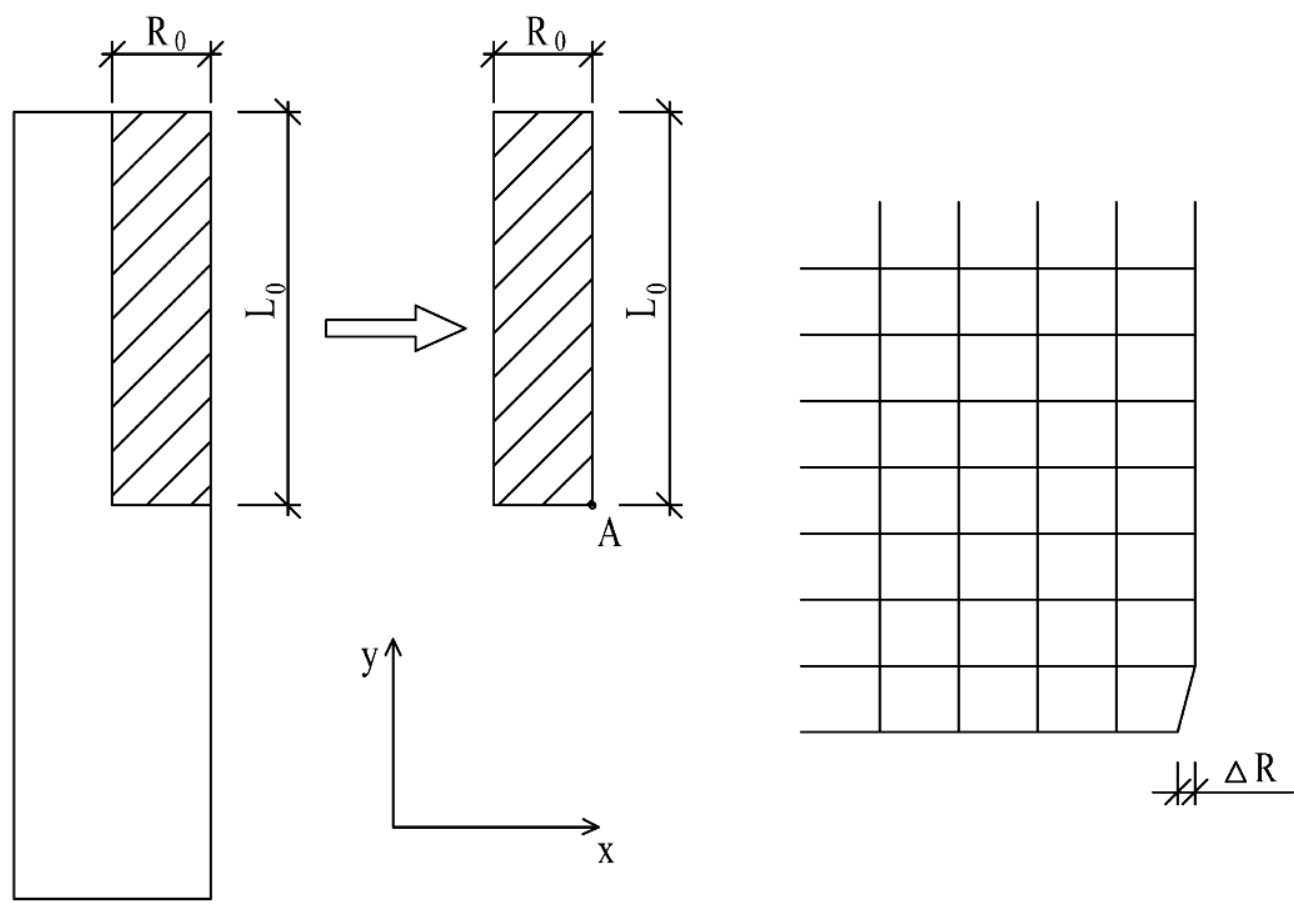

Figure 1: Geometry and imperfection of the specimen.

4-noded FEM elements [8] are used for discretization which divide the specimen into 1600 pieces called in ABAQUS system CAX4RT (Fig. 2a). Each node has 7 degree of freedom (6 mechanical and 1 thermal which is temperature). Mechanical boundary conditions are $u_{x}=0$ on the left side, $u_{y}=0$ on the bottom side and forced displacement $u_{y}=0.125 L_{0}$ applied to the top side (Fig. 2b). 


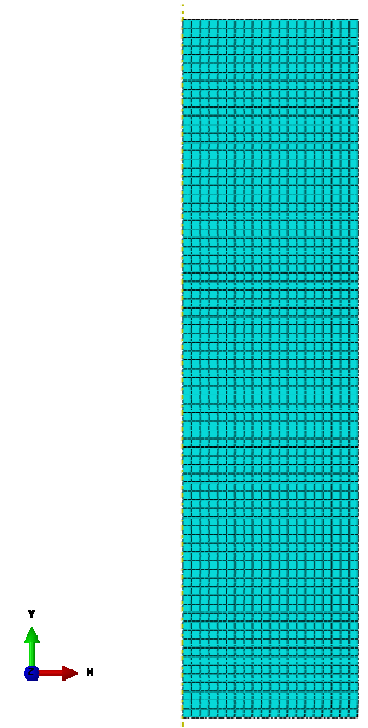

a)

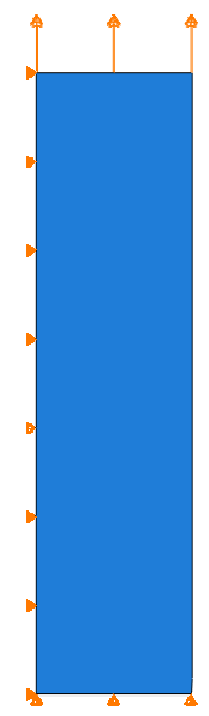

b)

Figure 2: FEM model (a) and thermal boundary conditions (b).

Initial temperatures are set equal to $T_{0}=20^{\circ} \mathrm{C}$ for all nodal points, while fire simulation is performed by setting surface temperatures equal to these calculated from the fire curve after ISO 834-1 statement (Fig. 3a). We set these temperature to the top and the right side of the specimen (Fig. 3b).

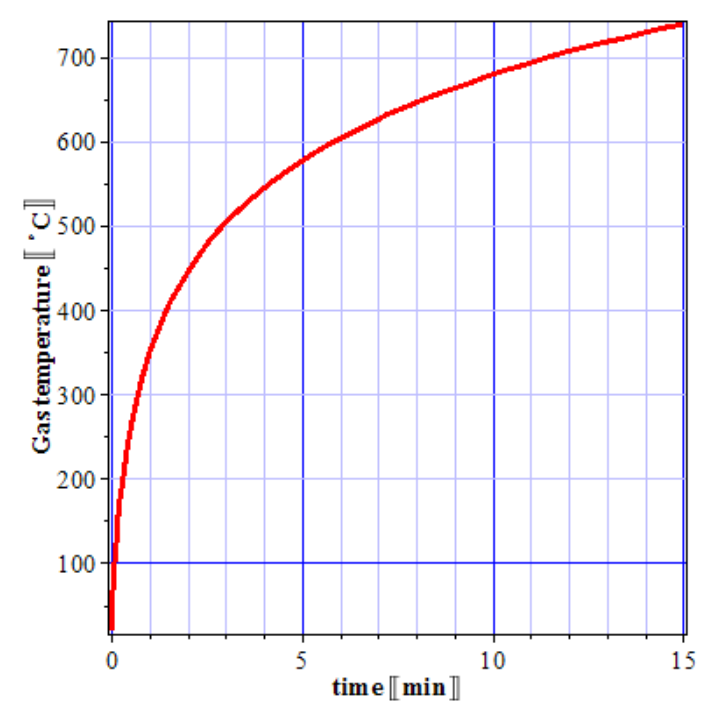

a)

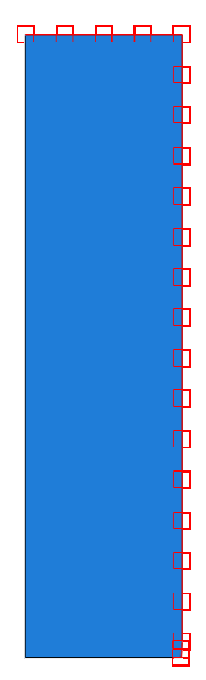

b)

Figure 3: Time-temperature curve (a) and thermal boundary conditions (b).

Steel properties as Young modulus, yield strength, conductivity, specific heat and coefficient of thermal elongation are highly temperature-dependent according to EN 1993-1-2. Fully coupled thermal-stress analysis is used where element temperature affects stress and displacements values. 11 tests were carried out with time period equal to 15 minutes for 11 different values of random variable which was gas temperature $b \equiv T_{s}$ in this case. Figures $4 \div 6$ show resulting Huber-Mises stress distribution on deformed specimen. As can be expected this distribution is not uniform. Moreover we can see precisely how necking rise. 


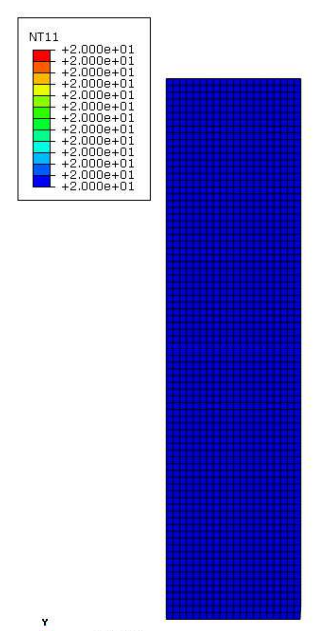

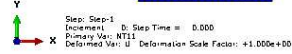

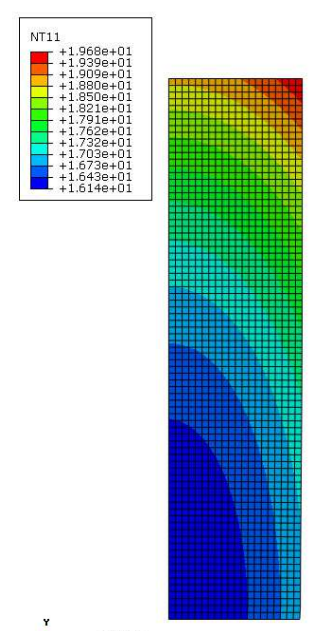

$\dot{L} \times x^{2}$

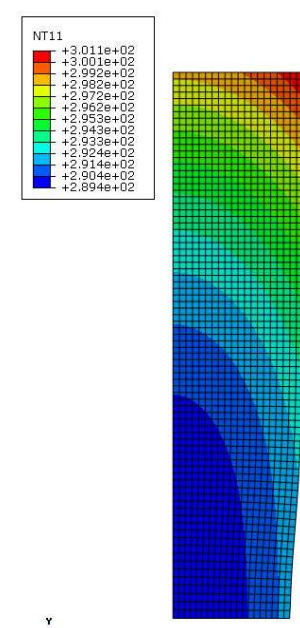

$\dot{L} \times x^{2}=$

Figure 4: Stress distribution at $t=0,60,300 \mathrm{~s}$.
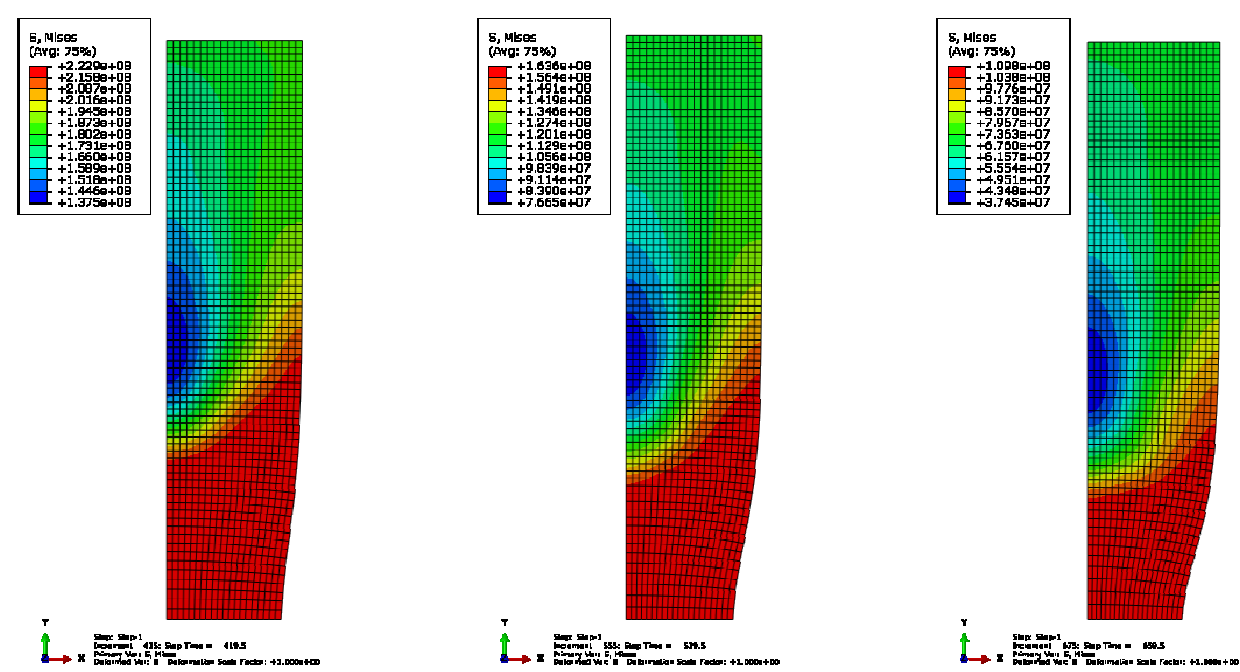

Figure 5: Stress distribution at $t=420,540,660 \mathrm{~s}$.
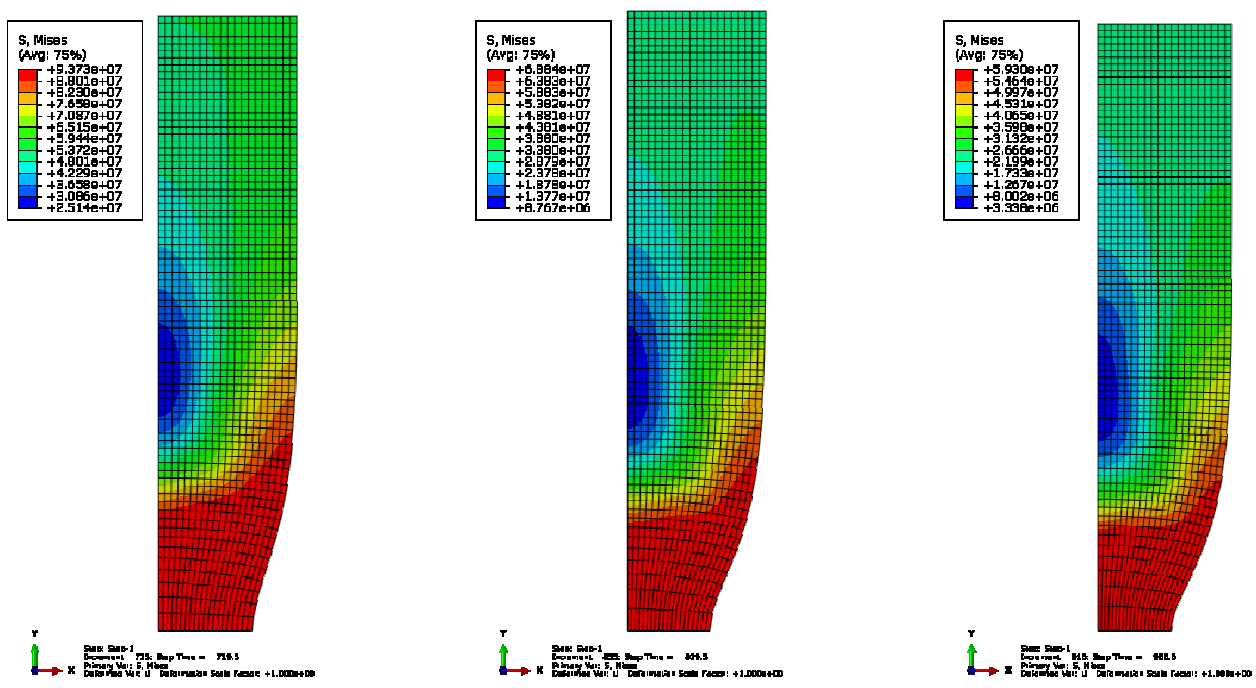

Figure 6: Stress distribution at $t=720,860,900 \mathrm{~s}$. 
19 polynomial response functions were performed to describe the response of the structure (Fig. 7a). Based on resulting stress values taken from FEM analysis results at an interval of 60 seconds we made time-dependent Huber-Mises diagram (Fig. 7b).

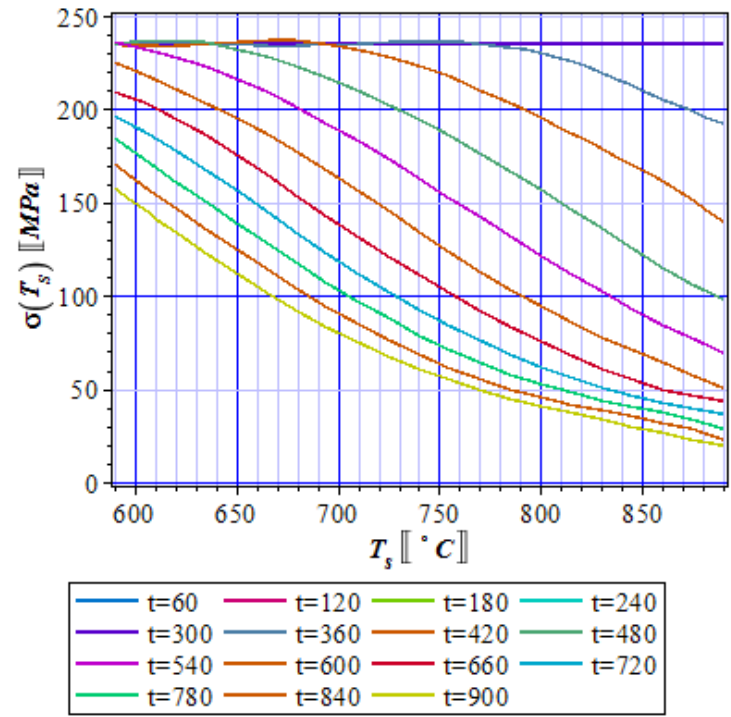

a)

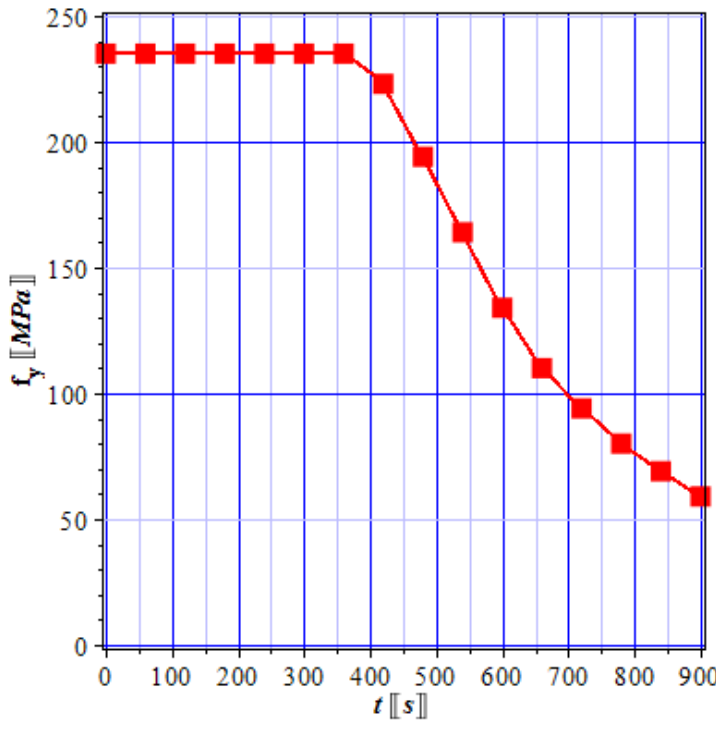

b)

Figure 7: Response functions (a) and resulting yield strength reductions (b).

Resulting gas temperature is also random variable in this case with its input coefficient of variation $\alpha\left(T_{s}\right)$ in the range of $[0.0,0.2]$. Figures $8 \div 9$ show expectation, coefficient of variation, skewness and kurtosis for extreme Huber-Mises stress in the point $A$ of the specimen (Fig. 1). The biggest fluctuations of expected values and coefficient of variation could be observed for $\alpha\left(T_{s}\right)=0.20$. Coefficients of variation are close to zero (small dispersion) for $\alpha\left(T_{s}\right) \leq 0.10$ and it means that values of stress are concentrated round its means.

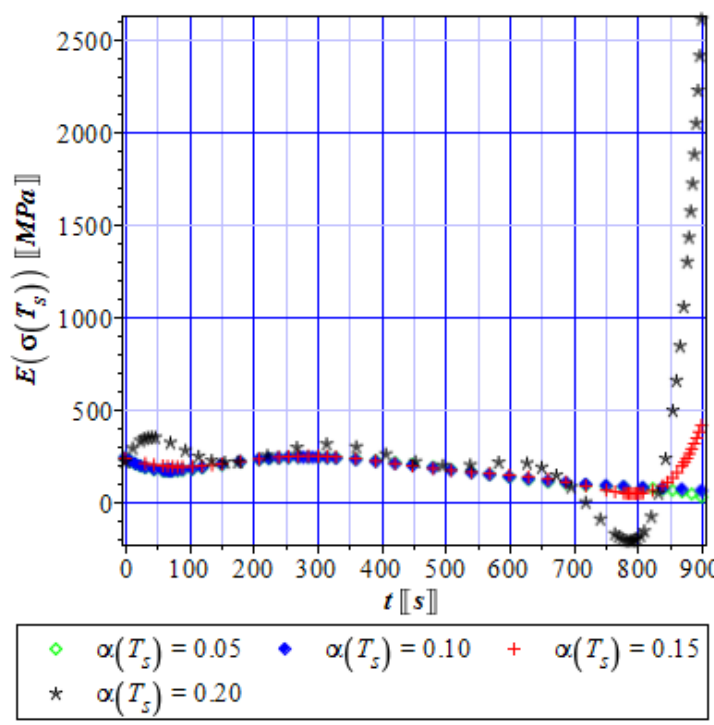

a)

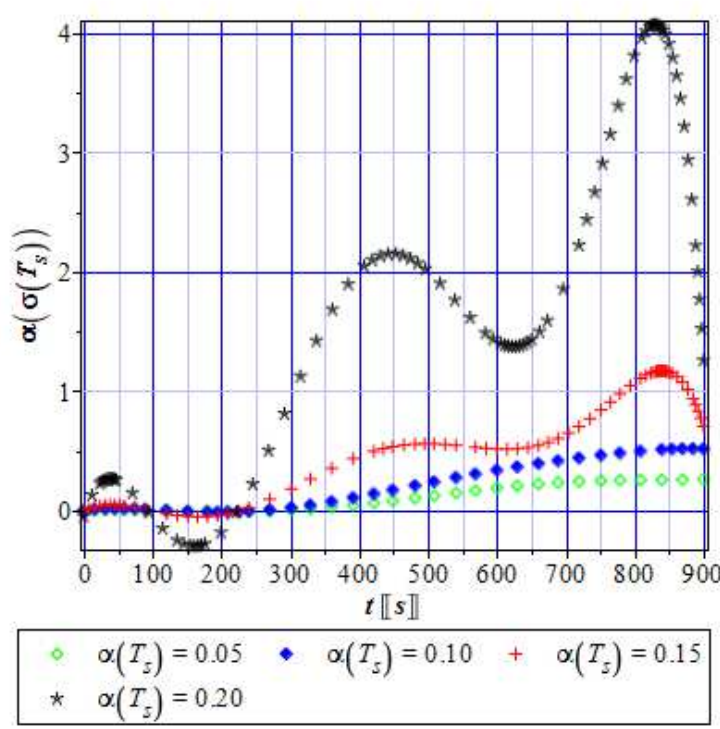

b)

Figure 8: Expectations (a) and coefficient of variation of stress (b). 


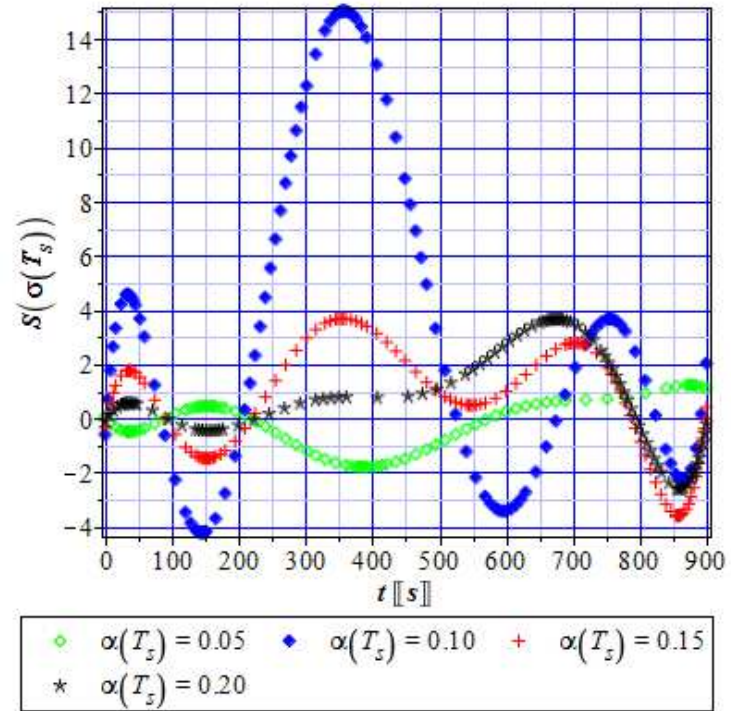

a)

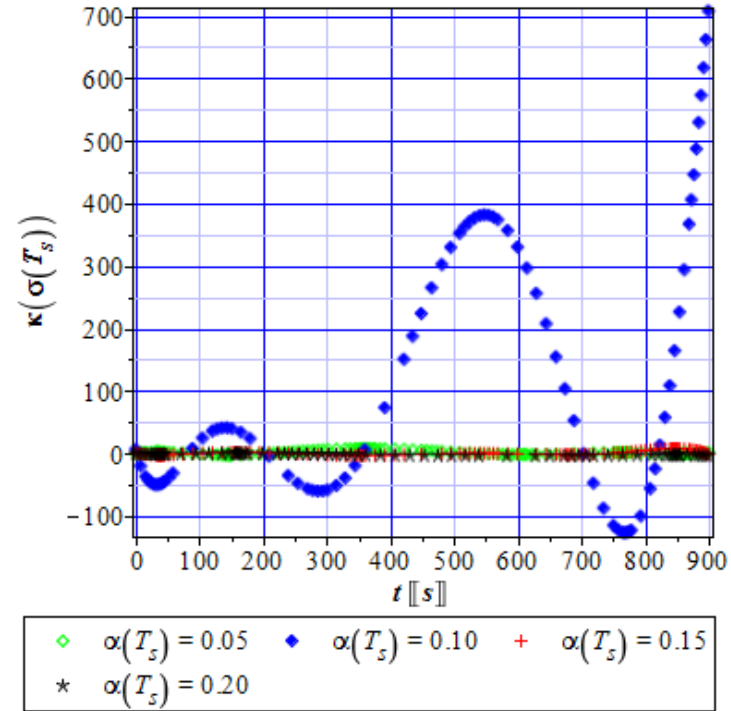

b)

Figure 9: Skewness (a) and kurtosis of stress (b).

Studying stress and kurtosis distributions we can see fluctuations for $\alpha\left(T_{s}\right)=0.10$. According to this plots we can conclude that Huber-Mises stress distribution is Gaussian when $\alpha\left(T_{s}\right) \leq 0.05$ because in this range skewness and kurtosis are close to zero.

This computation example has been made to determine the probabilistic characteristics for stress distribution under fire condition which is very often taken as non-Gaussian. This test illustrates the strength test cylindrical steel rod at elevated temperatures, the results of which enable determination of the yield point of the curve changes as a function of time.

\section{CONCLUSIONS}

Based on this computation we can say that probability distribution of reduced stress under fire condition is Gaussian only if we narrow the range for input coefficient of variation to $\alpha\left(T_{s}\right)<0.05$. Moreover we shown the common usage of FEM software ABAQUS and computer algebra system MAPLE which is crucial to use Stochastic Finite Element Method [9] (SFEM). We looked at the influence of polynomial degree [10] on the results of stochastic analysis. In such non-linear problems we have to use different functions to fit the response of the structure. The information about stress distribution obtained in this study can be use in the stochastic reliability analysis of the steel structures under fire according to both First and Second Reliability Method [3].

\section{REFERENCES}

[1] M. Kleiber, T.D. Hien, The Stochastic Perturbation Method for Computational Mechnics. Wiley, 1992.

[2] M. Kamiński, The Stochastic Perturbation Method for Computational Mechanics. Wiley, 2013. 
[3] M. Kamiński, M. Strąkowski, On the least squares stochastic finite element analysis of the steel skeletal towers exposed to the fire. Archives of Civil and Mechanical Engineering, 13, 242-253, 2013.

[4] M. Kamiński, P. Świta, Generalized stochastic finite element method in elastic stability problems. Computers and Structures, 89, 1241-1252, 2011.

[5] R. Putanowicz, J. Jaśkowiec, P. Pluciński, A. Stankiewicz, Finite element implementation of nonlinear thermo-elasticity as typical coupling of diffusion and momentum balance. Mechanics and Control, 32, 152-163, 2013.

[6] M. Kamiński, J. Szafran, Stochastic Analysis and reliability of the steel telecommunication towers. CMES: Computer Modeling in Engineering and Science, 83, 143-168, 2012.

[7] N. Aravas, On the numerical integration of a class of pressure-dependent plasticity models. International Journal for Numerical Methods in Engineering, 24, 1395-1416, 1987.

[8] O.C. Zienkiewicz, R.C. Taylor, The finite element method, Vol. I, 4th Edition. McGraw Hill, 1989.

[9] G. Stefanou, M. Papadrakakis, Stochastic finite element analysis of shells with combined random material and geometrical properties. Computer Methods in Applied Mechanics and Engineering, 193, 139-160, 2004.

[10] A. Björck, Numerical Methods for Least Squares Problems. SIAM, 1996. 